\title{
Desempenho motor de crianças de diferentes estágios maturacionais: análise biomecânica
}

\author{
Sebastião I. Lopes Melo \\ Roberta Gabriela O. Gatti \\ Roberta C. Detânico \\ Jansen A. Estrázulas \\ Ruy J. Krebs
}

https://doi.org/10.5628/rpcd.08.01.58

\author{
Laboratório de Biomecânica \\ Centro de Educação Física Fisioterapia e Desportos (CEFID) \\ Universidade do Estado de Santa Catarina UDESC \\ Brasil
}

\section{RESUMO}

Neste estudo descritivo exploratório avaliou-se as características biomecânicas do salto vertical de crianças de diferentes estágios maturacionais (inicial, elementar e maduro) segundo modelo de Gallahue (1989). Especificamente as variáveis entre os estágios foram caracterizadas e comparadas e foi verificada a contribuição de diferentes variáveis no desempenho.

Participaram 39 escolares, seleccionados pelo processo casual sistemático. Utilizou-se uma plataforma de força extensométrica a $900 \mathrm{~Hz}$, uma filmadora a $60 \mathrm{~Hz}$ e a matriz analítica de Gallahue (1989). Os dados dinâmicos foram normalizados pelo peso corporal, processados no sistema Peak Motus e filtrados via FFT Butherworth $4^{\mathrm{a}}$ ordem passa baixa, corte a $30 \mathrm{~Hz}$. Analisouse: pico e gradiente de propulsão, impulso vertical, impacto, tempo de vôo e ângulos de quadril e joelho na propulsão, vôo e queda. Utilizou-se a estatística descritiva, a ANOVA one-way com post-hoc de Scheffé e a regressão linear múltipla, com $\mathrm{p} \leq 0,05$. Verificou-se diferenças significativas entre os estágios no tempo de vôo e ângulo de quadril nas três fases do salto e ângulo de joelho no ápice do vôo. O impulso vertical foi a variável que teve maior contribuição na performance. As crianças do estágio maduro melhor coordenaram os segmentos corporais na execução do salto vertical.

Palavras-chave: salto vertical, desenvolvimento motor, crianças, biomecânica

\section{ABSTRACT \\ Motor development of children in different maturement stages:} biomechanics analysis

This descriptive exploratory study evaluated biomechanical characteristics of vertical jump of children in different maturation stages (initial, elementary and mature) according to Gallahue's model (1989).

Specifically, variables of different stages were characterized and compared and the contribution of these variables to the performance was verified. The sample was composed by 39 scholars, selected through a systematic casual sampling process. An extensometric force plate sampled at $900 \mathrm{~Hz}$, a $60 \mathrm{~Hz}$ video camera and Gallahue's Analytical Matrix (1989) were used. Dynamic data were normalized by body weight, processed through Peak Motus System and filtered by a fourthorder low-pass Butterworth filter with a $30 \mathrm{~Hz}$ cut-off frequency. Analyzed variables: peak and gradient of propulsion, vertical impulse, impact, flight time, hip and knee angles during propulsion, flight and landing phases. Data were analyzed through descriptive statistics, ANOVA one-way followed by Scheffé post-hoc test and also Multiple Linear Regression $(p \leq 0,05)$. One verified significant differences between the stages for flight time and hip angle during the three jump phases; and for knee angle during flight phase. Vertical impulse was the variable that most contributed to the performance. Children in mature stage presented better coordination of body segments during the vertical jump execution than children in other stages.

Key-words: vertical jump, motor development, children, biomechanics 


\section{INTRODUÇÃO}

O saltar é muito utilizado nas tarefas do cotidiano e nas diferentes tarefas motoras integrantes dos diferentes esportes de forma isolada ou combinada com outras habilidades. Trata-se de um padrão motor complexo que requer um recrutamento muscular num modo de contracção particular que faz suceder, em tempo extremamente breve, um ciclo alongamento-encurtamento do músculo(16). Portanto, requer execução com uma mecânica eficiente. Sabe-se que o desenvolvimento motor é uma área que utiliza o aprendizado motor, o controle motor, a fisiologia do exercício e a biomecânica e poucos são os estudos que utilizam as análises biomecânicas para avaliar as características de execução do salto de crianças. Ademais, várias pesquisas vêm sendo desenvolvidas $(9,13,3,15)$ tendo o salto vertical como meio de avaliação do potencial mecânico dos músculos dos membros inferiores, isto significa dizer que os estudos realizados visam à performance sendo, portanto direccionados, mais aos sujeitos envolvidos no esporte de competição, e não para avaliar o padrão motor do salto.

A literatura confirma que a melhor fase para o aprendizado e desenvolvimento motor ocorre na infância, pois o desenvolvimento motor segue uma determinada sequência de modificações nos movimentos que difere entre os indivíduos no instante da evolução, mas não na sequência que acontecem, porém em certos períodos da vida o indivíduo não pode atingir o aperfeiçoamento de suas capacidades se não for submetido a estímulos variados(6).

Assim, a partir da classificação das crianças em estágios de desenvolvimento motor, Gallahue propôs uma matriz de avaliação qualitativa, segundo a qual as crianças são observadas e em função de algumas características de posicionamento dos segmentos corporais, classificadas nos três estágios de desenvolvimento motor: inicial, elementar e maduro. Nem todos os padrões motores encaixam-se precisamente em uma progressão arbitrária de três estágios, entretanto, essa abordagem de Gallahue adapta-se, precisa e adequadamente, à sequência desenvolvimentista da maioria dos padrões motores fundamentais(8). Também, autores ${ }^{(8)}$ acrescentam que as crianças adquirem maior complexidade nos padrões motores após a mielinização do cerebelo, que ocorre entre 6 e
10 anos. E, a evolução do salto vertical ocorre aproximadamente dos 4 aos 11 anos, acrescentando que no processo de desenvolvimento deve-se explorar todas as possibilidades de movimento(1).

Diante do exposto e considerando que as pesquisas relativas ao salto vertical têm enfoque essencialmente voltadas para a performance esportiva ou como avaliação de capacidades físicas; considerando que os estudo dos padrões motores sob o foco da Teoria de Gallahue são essencialmente qualitativas, utilizandose uma matriz de análise de eventos motores através da observação visual; considerando que a biomecânica pode contribuir com indicadores quantitativos para serem adicionados aos elementos qualitativos; considerando a escassez de estudos de variáveis biomecânicas do salto vertical de crianças, justifica-se a realização deste estudo com objectivo geral de avaliar características biomecânicas do salto vertical de crianças de 4 a 12 anos. Mais especificamente, caracterizar e comparar as variáveis entre os estágios inicial, elementar e maduro segundo o modelo proposto por Gallahue(7) e verificar a contribuição de diferentes variáveis na execução do salto.

\section{MATERIAIS E MÉTODO}

Neste estudo descritivo exploratório foram analisadas as características biomecânicas do salto vertical de crianças na faixa etária de 4 a 12 anos. Participaram 39 estudantes da rede pública de ensino de Florianópolis-SC, Brasil, de ambos os sexos, com idade média de $8 \pm 2,13$ anos, seleccionados pelo processo casual sistemático, tendo como critério de exclusão a apresentação de alguma disfunção física aparente que impossibilitasse a prática de actividade física, diagnosticadas pelo médico da escola. $\mathrm{Na}$ aquisição dos dados dinâmicos utilizou-se uma plataforma de força extensométrica AMTI modelo OR6-5, nivelada ao solo; para os dados cinemáticos, uma câmera de vídeo Peak HSC-180, sincronizada com a plataforma e ambos integrados ao sistema Peak Motus e para classificação das crianças nos estágios motores, a matriz analítica proposta por Gallahue(7).

Após aprovação do Comitê de Ética em Pesquisa da UDESC foi feito contato com as diretorias das escolas e com os responsáveis pelas crianças, para obter autorizações respectivas. Preliminarmente à aquisição 
e após a autorização dos pais e o agendamento prévio das coletas, as crianças foram encaminhadas, pelos pesquisadores responsáveis, ao Laboratório, onde se adotou a seguinte seqüência de procedimentos: a) preenchimento da ficha de identificação pessoal e atribuição de código de identificação; b) adaptação dos sujeitos ao ambiente e aos equipamentos bem como com os pesquisadores; c) fixação dos marcadores reflexivos nos pontos anatômicos (têmporo-mandibular, ombro, cotovelo, punho, quadril, joelho e tornozelo), conforme modelo adaptado de Cavanagh e Rodgers(2) para servirem de referência para a determinação dos segmentos corporais e possibilitar os cálculos de ângulos entre os mesmos; d) posicionamento das crianças sobre a plataforma para a normalização dos dados pelo peso corporal; e) aquisição dos dados: os cinéticos a uma freqüência de amostragem de $900 \mathrm{~Hz}$ e os cinemáticos a $60 \mathrm{~Hz}$, sincronizados no sistema Peak Motus. Cada criança executou 3 saltos verticais válidos (impulsão e queda sobre a mesma plataforma de força e com ambos os pés). A imagem adquirida foi usada para classificar as crianças nos estágios motores segundo matriz analítica prospota por Gallahue(7) e para analisar os ângulos entre os segmentos corporais referentes as articulações do quadril e joelho em instantes específicas do salto (nos picos de propulsão e de queda e no ápice do vôo) identificados com base na curva da componente vertical da força de reação do solo (CVFRS). Para caracterizar e comparar as variáveis biomecânicas entre os estágios motores selecionouse as variáveis cinemáticas: ângulo de quadril no instante do pico de propulsão ( $\beta \mathrm{PP})$, ângulo de joelho no instante do pico de propulsão ( $\delta P P)$, ângulo de quadril no ápice do vôo ( $\beta A V)$, ângulo de joelho no ápice do vôo $(\delta \mathrm{AV})$, ângulo de quadril no instante do pico de queda ( $\beta \mathrm{PQ})$ e ângulo de joelho no instante do pico de queda $(\delta P Q)$. Os pontos de referências usados para marcar os ângulos foram digitalizados no sistema Peak Motus, conforme pode ser visualizado na Figura 1.
Da mesma forma, foram seleciondas as variáveis dinâmicas e espaço-temporais, que estão representadas na Figura 2, quais sejam: pico de propulsão (PP), gradiente de propulsão (GP), impulso vertical (IV), pico de queda (PQ), impulso de amortecimento (IA) e tempo de vôo (TV).

Para o processamento dos dados dinâmicos e cinemáticos utilizou-se o sistema Peak Motus, sendo que os dados dinâmicos foram processados via transformada rápida de Fourier (FFT) filtro Butterworth de $4^{\mathrm{a}}$ ordem passa baixa com frequência de corte de $30 \mathrm{~Hz}$. Para a caracterização das variáveis utilizou-se da estatística descritiva (média $(\overline{\mathrm{X}})$, desvio padrão (s) e coeficiente de variação (CV\%); para a comparação dessas entre os estágios foi utilizada a análise de variância com o teste post-hoc de Scheffé e para verificar o percentual de contribuição das variáveis na execução do salto fez-se a Regressão Linear Múltipla. O nível de confiança adotado para todas as análises foi de $95 \%$.

\section{RESULTADOS E DISCUSSÃO}

Para efeitos didácticos a fim de facilitar a compreensão do leitor, este capítulo foi organizado segundo os objectivos específicos do estudo. Assim, respondendo ao primeiro objectivo, segue a caracterização e comparação das variáveis dinâmicas e cinemáticas, cujos valores e diferenças entre os estágios podem ser visualizados nas Figuras 3, 4, 5, 6 e 7, a seguir.

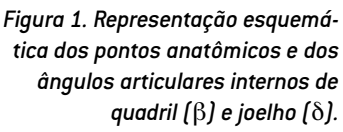
quadril $(\beta)$ e joelho $[\delta]$. 


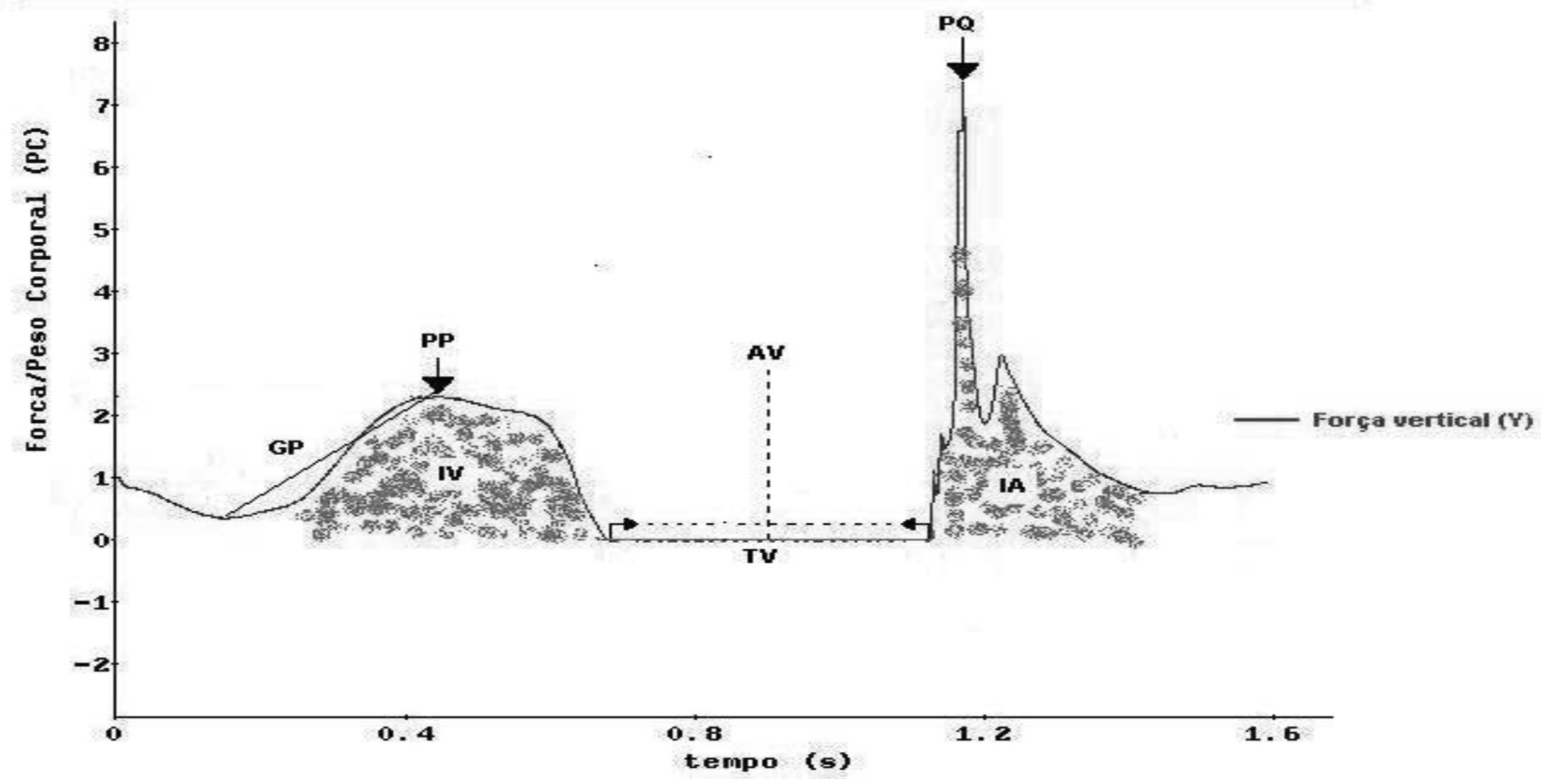

Figura 2. Representação da curva força $x$ tempo apresentando as variáveis dinâmicas e espaço-temporais utilizadas: PP - pico de propulsão, $T V$ - tempo de vôo, $P Q$ - pico de queda, GP - gradiente de propulsão, IV - impulso vertical, IA - impulso de amortecimento.

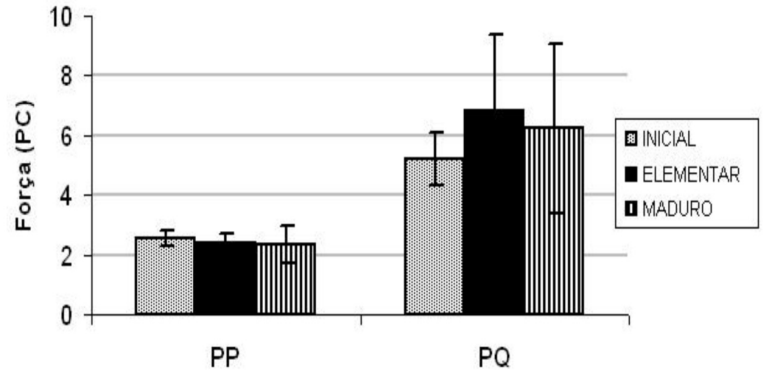

Figura 3. Picos de força na propulsão (PP) e na queda $(P Q)$.

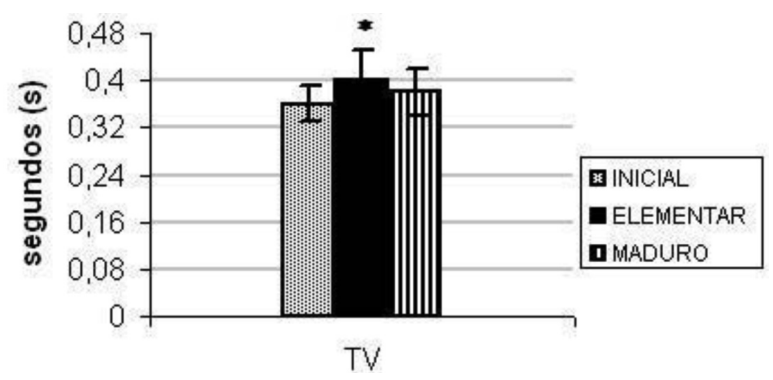

Figura 5. Tempo de vôo [TV]

* diferença estatisticamente significativa Elementar vs, Inicial e Maduro; $p<0.05$

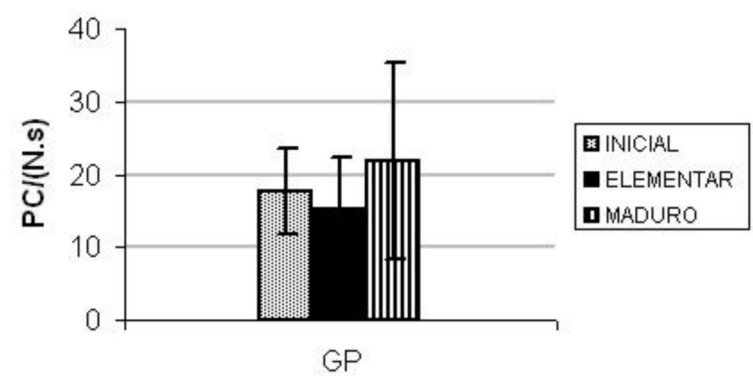

Figura 4. Gradiente de propulsão (GP)

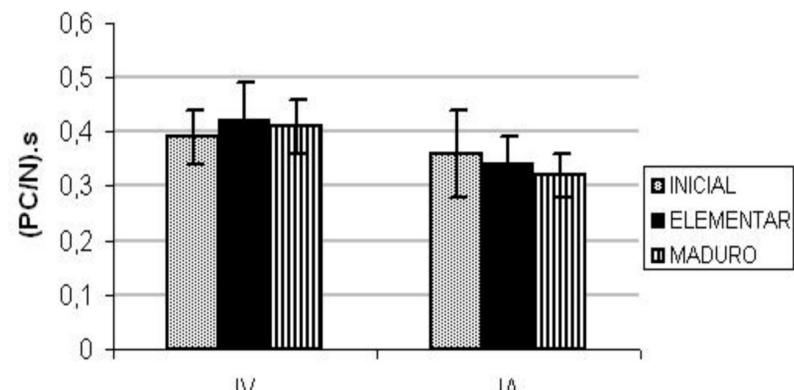

Figura 6. Impulso vertical na propulsão (IV) e impulso de amortecimento na aterrissagem (IA). 


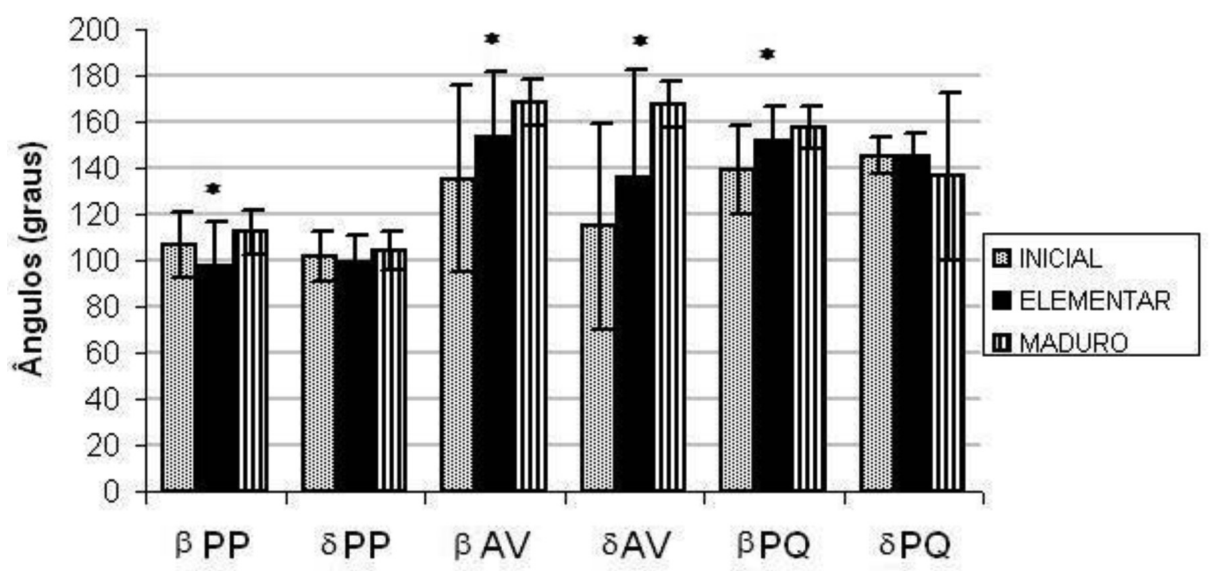

Figura 7.Variáveis angulares: ângulo de quadril no instante do pico de propulsão ( $\beta P P$ ), ângulo de joelho no instante do pico de propulsão ( $\delta P P$ ], ângulo de quadril no ápice do vôo ( $\beta A V$ ], ângulo de joelho no ápice do vôo [ $\delta A V$ ], ângulo de quadril no instante do pico de queda $(\beta P Q)$ e ângulo de joelho no instante do pico de queda $(\delta P Q)$.

* diferença estatisticamente significativa Elementar vs, Inicial e Maduro; $p<0.05$

Analisando os resultados contidos nas Figuras 3, 4, 5, 6 e 7 , constatou-se que das 12 variáveis estudadas 5 delas apresentaram diferenças significativas, em que pelo menos um dos estágios é diferente dos demais. Para verificar em quais estágios estavam tais diferenças, aplicou-se o teste de comparação múltipla de médias, análise "post-hoc" de Scheffé, cujos resultados estão dispostos no quadro observou-se que o estágio inicial sempre foi diferente do elementar ou do maduro para as cinco variáveis (Quadro 1).

Quadro 1. Resultados da comparação entre os diferentes estágios para as diferentes variáveis.

\begin{tabular}{lccccc}
\hline Variável & & $\overline{\mathrm{X}}$ & & $\begin{array}{c}\text { Diferenças } \\
\text { Verificadas }\end{array}$ & $\mathrm{P}$ \\
\hline & Inicial & Elementar & Maduro & & \\
\hline$\beta \mathrm{PP}\left({ }^{\circ}\right)$ & 106,47 & 97,65 & 112,23 & El. $\times$ Mad. & 0,05 \\
\hline $\operatorname{TV}(\mathrm{s})$ & 0,36 & 0,40 & 0,38 & In. $\times$ El. & 0,03 \\
\hline$\beta \mathrm{AV}\left(^{\circ}\right)$ & 135,29 & 153,26 & 168,44 & In. $\times$ Mad. & 0,01 \\
\hline$\delta A V\left(^{\circ}\right)$ & 114,64 & 135,34 & 167,51 & In. $\times$ Mad. & 0,01 \\
& & & & El. $\times$ Mad. & 0,01 \\
\hline$\beta P Q\left(^{\circ}\right)$ & 139,18 & 151,68 & 157,35 & In. $\times$ Mad. & 0,03 \\
\hline
\end{tabular}

A partir da interpretação do "post-hoc" de Scheffé para as variáveis acima, verificou-se que: a) Na variável ângulo de quadril no instante do pico de propulsão ( $\beta \mathrm{PP})$ o estágio elementar teve a menor média e o maduro a maior, ocorrendo diferenças significativas somente entre elementar $\mathrm{x}$ maduro $(p=0,05)$, com valores maiores para as crianças do estágio maduro;

b) Na variável tempo de vôo (TV) o estágio inicial teve a menor média e o elementar a maior, apresentando diferenças significativas somente entre inicial $x$ elementar $(p=0,03)$. Diferente do esperado, pois as maiores médias deveriam ocorrer no estágio maduro;

c) Na variável ângulo de quadril no ápice do vôo $(\beta A V)$ o estágio inicial apresentou a maior flexão e o maduro a menor, ocorrendo diferenças significativas apenas entre inicial $x$ maduro $(p=0,01)$, indicando uma melhor utilização dessa articulação pelas crianças do estágio maduro;

d) Na variável ângulo de joelho no ápice do vôo $(\delta \mathrm{AV})$ as crianças do estágio elementar apresentaram a maior flexão e as do maduro a menor, com diferenças significativas entre inicial $x$ maduro $(p=0,01)$ e elementar $\mathrm{x}$ maduro $(\mathrm{p}=0,01)$, mas não entre inicial $\mathrm{x}$ elementar. Isso significa que, conforme o esperado para essa fase do salto, as crianças do estágio maduro seguido pelas do elementar se mostraram em maior extensão do corpo durante o vôo que as do inicial; 
e) Na variável ângulo de quadril no instante do pico de queda $(\beta P Q)$ as crianças do estágio inicial apresentaram o quadril mais flectido e as crianças do estágio maduro mais estendido, apresentando diferenças significativas somente entre inicial $\mathrm{x}$ maduro $(p=0,03)$, e o maduro sendo diferente dos demais. Possivelmente esse fato tenha ocorrido devido às crianças do estágio inicial terem assumido a posição do corpo em maior flexão durante o vôo e consequentemente caíram em maior flexão que as crianças dos demais estágios.

Os resultados destas comparações indicam que a melhora da técnica do salto, que depende também do posicionamento dos segmentos corporais, representados pelos ângulos das articulações do corpo, aconteceu predominantemente do estágio inicial para o maduro.

Numa visão global, na análise dos resultados dispostos nas Figuras 3, 4, 5, 6 e 7 e no Quadro 1, constatase que para a maioria das variáveis os valores médios obtidos nos três estágios foram semelhantes, entretanto para algumas variáveis os valores foram bastante díspares, sendo que o estágio inicial apresentou as maiores médias em 3 delas (PP, IA e $\delta P Q$ ), o estágio elementar em outras 3 (IV, TV e PQ) e o maduro nas 6 restantes (GP, $\beta P P, \delta P P, \beta A V, \delta A V$ e $\beta P Q$ ).

$\mathrm{Na}$ caracterização dos resultados das diferentes variáveis do salto fez-se a análise da homogeneidade e o confronto com a literatura. Quanto à homogeneidade observa-se que a variabilidade para maioria das variáveis foi considerada de média (11\% a $20 \%)$ a alta (acima de $30 \%)^{(10)}$; contudo, a amplitude variou entre $5,42 \%$ e $48,22 \%$. Tais índices podem ser explicados ao se considerar a ampla faixa etária, no intuito de contemplar os três estágios maturacionais, bem como pela estratégia individual adoptadas pelas crianças na execução da tarefa motora proposta. Por outro lado, esta variabilidade pode ser considerada normal, pois pode ser influenciada por diferentes aspectos da constituição física influenciam a capacidade de desempenho motor (5).

Considerando ser um estudo exploratório, o confronto com a literatura foi prejudicado. Desta forma, tais resultados, quando possível, foram confrontados com a literatura existente, mas contribuem com o estabelecimento de valores referência para estudos posteriores, disponibilizando um banco de dados com características de diferentes variáveis biomecânicas do movimento do salto vertical de crianças. Na variável pico de propulsão (PP) verificou-se que as crianças do estágio inicial aplicaram, em média, mais força $(2,55 \pm 0,25 \mathrm{PC})$ para saltar seguido das crianças do estágio elementar $(2,39 \pm 0,34 \mathrm{PC})$ e por fim as crianças do estágio maduro $(2,35 \pm 0,61 \mathrm{PC})$. Estes resultados dos valores de PP encontrados nesse estudo diferem do resultado obtido em outro estudo(11), que ao estudar o salto horizontal de crianças no estágio elementar e maduro, encontrou valores de $\mathrm{PP}$ para o estágio elementar de 2,23 $\pm 0,31 \mathrm{PC}$ e para o estágio maduro de $2,18 \pm 0,22 \mathrm{PC}$. Verifica-se que os valores de PP foram maiores na realização do salto vertical que no horizontal para as crianças de ambos os estágios, característica esta justificada pelo objectivo diferenciado dos saltos: o vertical a altura e o horizontal a distância.

No instante do pico de queda (PQ) os valores de impactos, para as crianças do estágio elementar $(6,80 \pm 2,58 \mathrm{PC})$ e para as do maduro $(6,23 \pm 2,83 \mathrm{PC})$, foram maiores comparados a outro estudo realizado com adolescentes não-atletas para a mesma tarefa(4), em que obtiveram valores médios de impacto de $5,55 \pm 1,26 \mathrm{PC}$. Contudo, para o estágio inicial $(5,21 \pm 0,87 \mathrm{PC})$ os resultados deste estudo foram ligeiramente inferiores.

E, de acordo com os achados no estudo de Fantini e Menzel(4), valores de impactos no instante do pico de queda maiores que 5,0 PC representam um aumento do risco de lesão, sendo que o flexionamento no primeiro contacto com o solo e a flexão plantar do pé possibilitam uma técnica de aterrissagem mais aprimorada. Esta atitude motora favorece a redução da força de colisão, pela dissipação das forças em diferentes direcções.

Em complemento, a variável impulso de amortecimento refere-se à capacidade de suavizar a queda $\mathrm{e}$ minimizar o impacto, através de um maior tempo para restabelecer o equilíbrio ortostático. Observando-se os dados verificam-se valores médios muito próximos para os três estágios maturacionais, sendo que o estágio inicial apresentou os valores médios ligeiramente maiores $(0,36 \pm 0,08 \mathrm{PC} / \mathrm{N} . \mathrm{s})$, seguidos pelo estágio elementar $(0,34 \pm 0,05 \mathrm{PC} / \mathrm{N} . \mathrm{s})$ e maduro $(0,32 \pm 0,04 \mathrm{PC} / \mathrm{N} . \mathrm{s})$. Tais resultados podem ser explicados pelo modo de execução do 


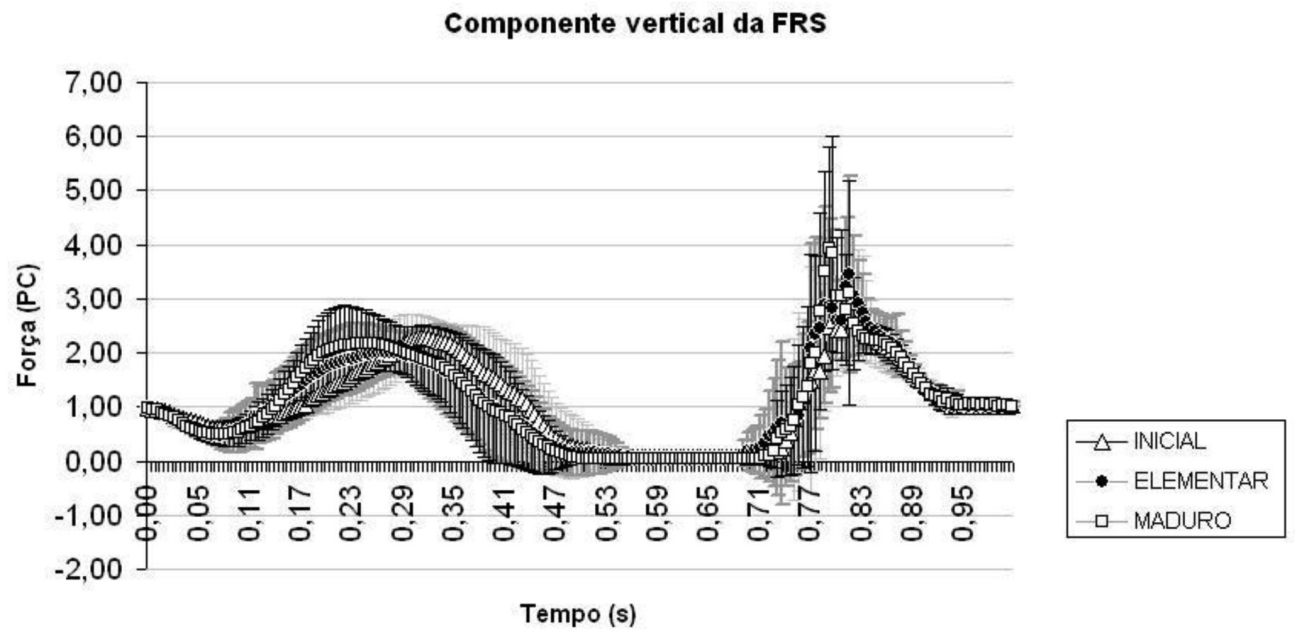

Figura 8. Representação da curva média e do desvio padrão da componente vertical da FRS para os três estágios de maturação sobrepostas.

salto vertical pelas crianças do estágio inicial que utilizam os segmentos corporais de forma mais flectida, apesar de ainda constituir um movimento pouco consistente, conforme pode-se confirmar pelos valores de desvio padrão para os três estágios. Tais resultados dinâmicos podem ser melhor visualizados na Figura 8.

Com relação aos valores de ângulos de quadril ( $\beta P P)$ e joelho (SPP) no instante de maior força de propulsão (PP), apresentados na Figura 7 e 9a, constata-se que nesse instante os segmentos analisados no estágio elementar apresentaram maior flexão

$\left(97,65 \pm 18,67^{\circ}\right.$ e $\left.99,32 \pm 11,55^{\circ}\right)$ e o estágio maduro uma menor flexão $\left(112,23 \pm 9,85^{\circ}\right.$ e $\left.104,03 \pm 8,30^{\circ}\right)$. Isso indica que as crianças do estágio elementar agacharam mais, o que parece ser favorável para o alcance de maiores alturas de salto, conforme disposto no modelo de Gallahue (7).

No ponto mais alto da trajetória do salto constatouse uma maior flexão de quadril ( $\beta \mathrm{AV})$ e joelho $(\delta \mathrm{AV})$ nas crianças do estágio inicial $\left(135,29 \pm 40,45^{\circ} \mathrm{e}\right.$ $114,64 \pm 44,94^{\circ}$ ) e uma maior extensão nas crianças do estágio maduro, com valores médios de $168,44 \pm 9,98^{\circ}$ e $167,51 \pm 9,80^{\circ}$, para quadril e joelho, respectivamente. Condição esta confirmada no modelo de Gallahue( ${ }^{(8)}$, cujo estágio inicial é caracterizado pela extensão insuficiente do corpo, o elementar semi-extensão e o maduro; extensão total do corpo durante o vôo do salto vertical.

No instante de maior valor do impacto verificou-se maior flexão para o quadril no estágio inicial $\left(135,18 \pm 19,39^{\circ}\right)$ e maior extensão no quadril no estágio maduro $\left(157,35 \pm 9,33^{\circ}\right)$. Ao contrário aconteceu com o ângulo de joelho cujas crianças do estágio maduro apresentaram-se em maior flexão $\left(136,51 \pm 36,13^{\circ}\right)$ e o estágio elementar em maior extensão $\left(145,14 \pm 9,49^{\circ}\right)$. Os valores de ângulos no instante do pico de força na queda (PQ) demonstraram pouco flexionamento das articulações para os três estágios, o que seria importante para amenizar o valor de impacto na aterrissagem. Essa observação pode ajudar a compreender o menor valor de impacto do estágio maduro comparado com o elementar, visto que o estágio maduro flexionou mais o joelho nesse instante, propiciando um melhor amortecimento das forças reactivas na fase de queda. E ainda, visto que as crianças do estágio elementar permaneceram maior tempo no ar, somado aos altos picos de queda atingidos por este grupo e à pouca flexão de joelho no instante de queda, pode-se atribuir que tenham também atingido maiores alturas.

O comportamento dos ângulos de quadril $(\beta)$ e de

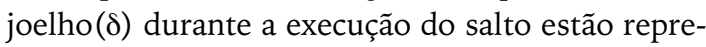
sentados na Figura 9a e 9b, nas quais é possível identificar as diferenças entre os estágios maturacionais, com o estágio elementar apresentando valores 

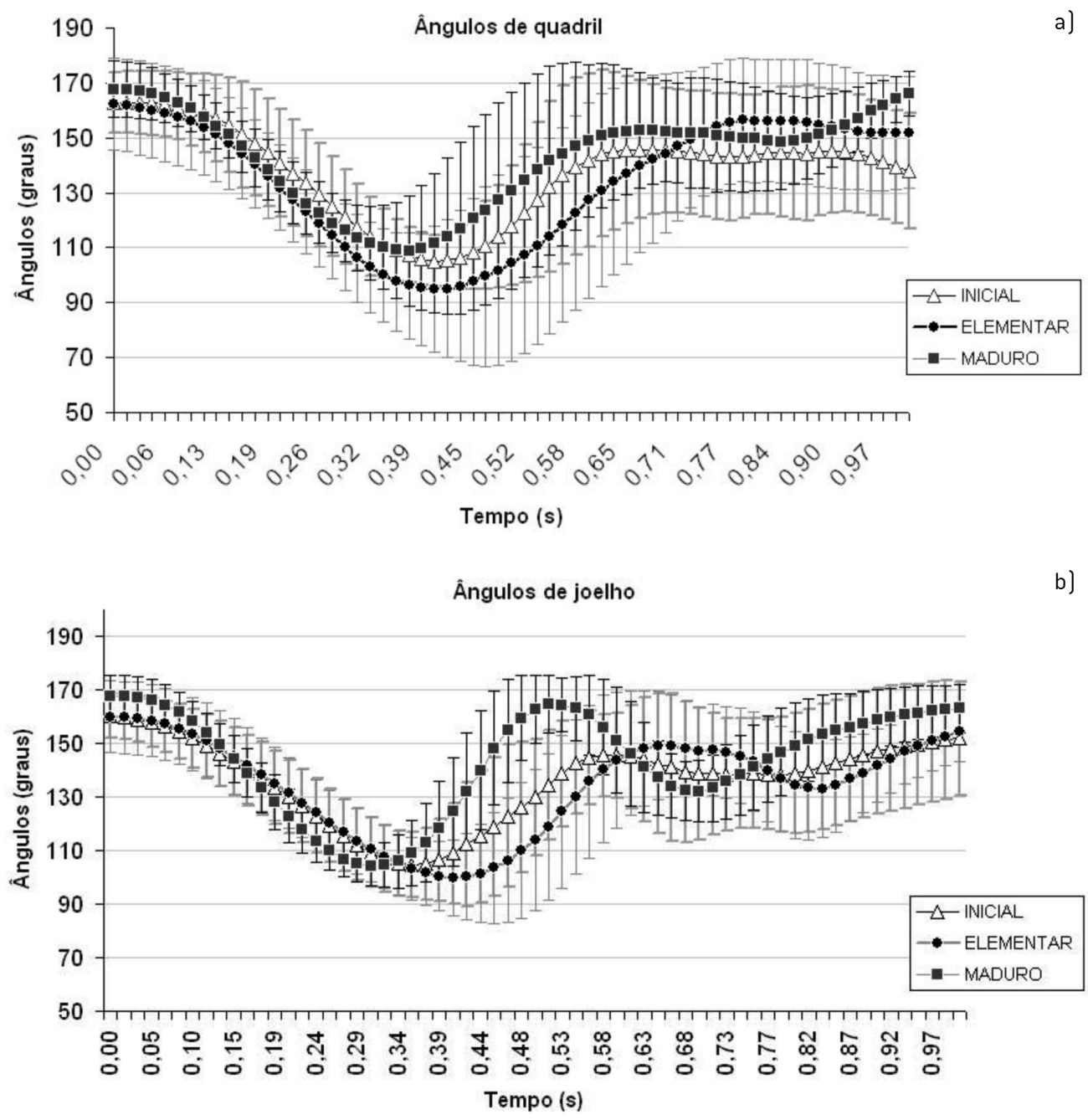

Figura 9. Representação do comportamento angular do quadril (a) e joelho (b)

dos estágios inicial, elementar e maduro, em toda a execução do salto.

de ângulos intermediáros, entre os ângulos do estágio inicial e maduro para os dois segmentos observados. Mesmo as curvas apresentando um mesmo formato para os três estágios, estas diferem, principalmente, em magnitude.

Dada à importância da utilização do corpo como um todo na execução da tarefa motora do salto, o fato das crianças do estágio maduro terem utilizado melhor os segmentos corporais pode ter influenciado na quantidade de força aplicada durante a propulsão sem que isso interferisse significativamente nos valores de força e no tempo de vôo, visto que não houve diferenças significativas para pico de propulsão entre os estágios e para tempo de vôo entre inicial e elementar. Esses achados se aproximam dos resultados de outros estudos(14), os quais afirmam que a máxima altura atingida no salto vertical, além de ser dependente da força muscular, pode ser optimizada pela máxima extensão de joelho e principalmente através da coordenação de quadril-joelho. 
Pode-se afirmar, assim, que a coordenação dos movimentos e a performance do salto vertical sofrem influência do tipo de estímulo de treinamento(12). Ainda com relação ao posicionamento dos segmentos corporais, constatou-se que na maioria das situações, foram as crianças do estágio inicial que apresentaram menores médias para as variáveis de ângulos e as crianças do estágio maduro as maiores médias, indicando que as crianças do estágio inicial flexionaram mais as articulações dos membros inferiores durante a propulsão. Este resultado permite afirmar que a evolução do salto vertical ocorre do estágio inicial para o maduro, verificando-se no estágio maduro maior aproximação da utilização do corpo durante a propulsão e queda, como modelo de Gallahue( ${ }^{(8)}$.

O segundo objetivo específico do estudo foi verificar a contribuição das diferentes variáveis biomecânicas na performance do salto vertical. Para tal aplicou-se o teste de regressão linear múltipla, do tipo "enter", que faz o cálculo da regressão sem ignorar nenhuma variável. Com este cálculo, pode-se estar calculando variáveis pouco representativas para o desempenho do salto horizontal, por isso aplicou-se novamente esta regressão para as variáveis que acusaram significância no primeiro teste. Este procedimento visa à retirada de informações chamadas de "lixo".

Desse modo, os resultados possibilitaram a composição da seguinte equação:

$\mathrm{Y}=\mathrm{a} \pm\left(\mathrm{X}_{1}+\mathrm{X}_{2}+\mathrm{X}_{3}+\mathrm{X}_{4+} \mathrm{X}_{5}\right)$, onde: $\mathrm{Y}=$ altura alcançada; $\mathrm{a}=$ intercepto; $\mathrm{X}_{1}=$ pico de propulsão (PP); $\mathrm{X}_{2}=$ impulso vertical; (IV); $\mathrm{X}_{3}=$ ângulo de quadril na propulsão $(\beta P P) ; X_{4}=$ ângulo de joelho na propulsão ( $(\mathrm{PP}) ; \mathrm{X}_{5}=$ ângulo de quadril no vôo ( $\beta \mathrm{AV}$ )

$$
\begin{gathered}
\text { PERFORMANCE }=27,5 \pm(0,095 \times \mathrm{PP}+0,45 \times \mathrm{IV} \\
-0,195 \times \beta \mathrm{PP}-0,099 \times \delta \mathrm{PP}+0,233 \times \beta A V) \\
\text { R-quadrado ajustado }=0,12
\end{gathered}
$$

Interpretando os resultados da Regressão através da equação anteriormente apresentada constatou-se que apenas a variável impulso vertical contribuiu significativamente para o tempo de vôo $(\mathrm{p}=0,009)$ com $45,0 \%$. O que significa dizer que para cada segundo de permanência no ar, o impulso vertical contribuiu positivamente com $45,0 \%$.
Para cada segundo de permanência no ar a variável pico de propulsão contribuiu positivamente com $9,5 \%$, a variável ângulo de quadril na propulsão contribuiu negativamente com $19,5 \%$, a variável ângulo joelho na propulsão teve contribuição negativa de $9,9 \%$ e a variável ângulo de quadril no vôo contribuiu positivamente com $23,3 \%$.

De um modo geral, os resultados da regressão linear múltipla de todas as variáveis do estudo para as 39 crianças, considerando-se a variável tempo de vôo como dependente das variáveis independentes (cinéticas e cinemáticas), constatou-se que apenas 12,0\% da variabilidade do tempo de permanência no ar pode ser explicado pelo efeito da variabilidade das variáveis avaliadas nesse modelo: impulso vertical (IV), pico de propulsão (PP), ângulo de quadril $(\beta P P)$ e joelho (SPP) na propulsão e ângulo de quadril no vôo ( $\beta A V)$.

Também o baixo valor do $R$ square ajustado pode ser explicado primeiramente pelo reduzido número de sujeitos da amostra e em virtude da ampla faixa etária em que as crianças se encontram, consequentemente em diferentes estágios maturacionais para a tarefa motora do salto vertical. Há de se considerar ainda a alta variação do movimento de uma execução para outra, visto que não são atletas, mas sim crianças em desenvolvimento, cujos movimentos ainda são executados de forma inconsistente, especialemente em tarefas motoras complexas como o salto vertical.

No que se refere à contribuição negativa dos ângulos de quadril e joelho na fase propulsiva, considerando os altos valores angulares obtidos, acredita-se que para que essa contribuição fosse positiva as crianças deveriam ter fletido mais tais segmentos nessa fase para alcançar melhor performance no salto, o que vai ao encontro da teoria de Gallahue(7).

\section{CONCLUSÕES}

A partir dos resultados obtidos, do referencial teórico consultado e das limitações presente estudo, pode se tecer as seguintes considerações:

As crianças do estágio maduro, conforme o esperado, coordenaram melhor os movimentos corporais durante toda a execução do movimento, o que favoreceu na aplicação das forças, pois apresentaram melhor disposição para activar o ciclo alongamento- 
encurtamento das fibras musculares envolvidas, principalmente na fase de propulsão do salto vertical. Cada criança apresenta sua própria estratégia de execução do salto vertical independente do estágio motor e que o maior incremento do salto não depende somente de força física, mas também da utilização dos segmentos corporais, explicado pela alta variabilidade das diferentes variáveis.

A partir do modelo utilizado, a variável impulso vertical foi a que melhor contribuiu positivamente para a performance da tarefa motora proposta.

Nesse contexto, acredita-se que tais informações poderão auxiliar o profissional da Educação Física na compreensão da técnica do salto vertical das crianças, o que será benéfico no que diz respeito ao aprendizado do movimento enquanto actividade lúdica, à performance desse movimento para a prática desportiva como também na minimização dos efeitos das colisões sobre o aparelho locomotor.

\section{REFERÊNCIAS BIBLIOGRÁFICAS}

1. Calomarde AB, Calomarde RB, Asensio SF (2003). Las habilidades motoras básicas. 2003 [cited $2003 \mathrm{Apr}$ 2005]; Available from:

http://www.ul.ie/ pess/research/motordev/vertical\%20ju mp.htm

2. Cavanagh PR, Rodgers MN (1987). The arch index: a useful measure form footprints. J Biomech 20(3):547-51.

3. Davisa DS, Briscoeb DA, Markowiski CT, Savilled SE, Taylore CJ (2003). Physical characteristicas that predict vertical jump performance in recreational male athletes. Physical Therapy in Sport 4:167-74.

4. Fantini C, Menzel H (2001). Análise de impactos em aterrissagens após saltos máximos em diferentes grupos de atletas e não-atletas. 9th Congresso Brasileiro de Biomecânica; Gramado: Escola de Educação Física da UFRGS - Sociedade Brasileira de Biomecânica 2001, p. 8993.

5. Ferreira M, Böhme MTS. (1998). Diferenças sexuais no desempenho motor de crianças: influência da adiposidade corporal. Revista Paulista de Educação Física 2:181-92.

6. Ferreira Neto CA (1995). Motricidade e jogo na infância.

7. Gallahue D (1989). Understanding motor development: infants, children, adolescents. 2 ed. Indiane: Benchmark.

8. Gallahue D, Ozmun JC (2001). Compreendendo o desenvolvimento motor: bebês, crianças, adolescentes e adultos. São Paulo: Phorte.

9. Garcia MAC, Massimiliani R, Oliveira LF, D'angelo MD (1993).Variáveis biomecânicas do salto vertical em atletas de voleibol. 5th Congresso Brasileiro de Biomecânica; Santa Maria: Sociedade Brasileira de Biomecânica, 75-8.

10. Gomes FP (1990). Curso de estatística experimental. 13 ed. Piracicaba: Degaspari.

11. Gress FAG (2004). Características dinâmicas do salto horizontal de crianças com idade entre 6 e 9 anos. Florianópolis: Mestrado em Ciências do Movimento Humano da Universidade do Estado de Santa Catarina.

12. Rodacki ALF, Fowler NE (2002). The specificity of two training programmes on vertical jump coordination. Braz J Biomech 3(4):27-35.

13. Spägele T, Kistner A, Gollhofer A (1999). A multi-phase optimal control technique for the simulation of a human vertical jump. J Biomech 32(1):87-91.

14. Tomioka M, Owings TM, Grabiner MD (2001). Lower extremity strength and coordination are independent contributors to maximum vertical jump height. J Appl Biomech 17(3):181-7.

15. Vanrenterghem J, Lees A, Lenoir M, Aerts P, De Clercq D (2004). Performing the verticl jump: movement adaptations for submaximal jumping. Hum Mov Sci 22:713-27.

16. Viel E. (2001). A marcha humana, a corrida e o salto biomecânica, investigações, normas e disfunções. São Paulo: Manole.

\section{CORRESPONDÊNCIA}

\section{Sebastião Iberes Lopes Melo}

Rua Paschoal Simone, 358 - Coqueiros

Florianópolis, SC, Brasil

CEP 88080-350

E-mail:d2silm@udesc.br e d6rcd@udesc.br 